\title{
A finiteness theorem for a dynamical class of entire functions
}

\author{
LISA R. GOLDBERG AND LINDA KEEN \\ C.U.N.Y., 33 West 42 Street, New York, NY 10036, USA
}

(Received 30 April 1984 and revised 14 January 1985)

\begin{abstract}
We define a class $\Sigma$ of entire functions whose covering properties are similar to those of rational maps. The set $\Sigma$ is closed under composition of functions, and we show that when regarded as dynamical systems of the plane, the elements of $\Sigma$ share many properties with rational maps. In particular, they have finite dimensional spaces of quasiconformal deformations, and they contain no wandering domains in their stable sets.
\end{abstract}

\section{0: Introduction}

Motivated by recent activity in the theory of dynamics of rational maps, we consider the problem of iterating a transcendental entire map of the plane.

Early in the 1900s Fatou and Julia independently developed a theory of rational maps as dynamical systems. Under iteration of a rational map $R$, the Riemann sphere decomposes into two completely invariant sets: a stable region, on which the behaviour of $R$ is either dissipative or elliptic; and an unstable region, on which $R$ is chaotic.

In 1926, Fatou [7] observed that this decomposition has an analogue for transcendental entire maps. He then showed that, while the unstable set of an entire map shares many of the qualitative features of the unstable set of a rational map, the stable properties can be quite different.

In [13] Sullivan proved a 'no wandering domain' theorem for rational maps that, combined with the work of Fatou and Julia, gives a complete classification of the behaviour of $R$ on its stable set. This classification depends heavily on the covering properties of $R$, namely:

(1) There is a strong relationship between the stable phenomena and the asymptotic behaviour of the values at which $R$ fails to be a covering map.

(2) The only values at which $R$ fails to be a covering map are the critical values. Since the covering properties of a transcendental entire map $E$ are more complicated than those of $R$, the classification theorem does not carry over.

In this article, we define a class $\Sigma$ of entire maps of finite type [see $\S 1$ ] whose covering properties are close to the covering properties of a rational map. For this class we prove an essential finiteness theorem:

THEOREM 3.1. If $E: \mathbb{C} \rightarrow \mathbb{C}$ has finite type, then $E$ has a finite dimensional space of quasiconformal deformations. 
This theorem, together with the good covering properties of $E \in \Sigma$ allows us to study stable behaviour of finite type entire maps, and in $\S 4$ we prove:

THEOREM 4.2. If $E: \mathbb{C} \rightarrow \mathbb{C}$ has finite type, then $E$ has no wandering domains.

It can further be shown that the classification of stable regions for rational maps can be modified to give a classification of stable behaviour for finite type entire maps [9].

The paper is arranged as follows. In $\S 1$, we define our class $\Sigma$, and determine some of its basic properties. $\$ 2$ contains technical definitions and standard theorems needed in the proof of theorem 3.1 , which constitutes $\S 3 . \S 4$ is devoted to dynamical applications of theorem 3.1 .

The authors wish to thank Dennis Sullivan for introducing this subject to them and for his encouragement in this project. They also wish to thank Michael Shub, Focurt Tangerman and Bob Devaney for general mathematical discussions of the material. We further wish to thank the referee for his general comments and his simplification of the proof of theorem 4.2. Finally, we wish to acknowledge A. Eremenko and M. Ljubic who have announced an independent proof of similar results.

\section{Section 1}

Throughout this paper, $\mathbb{C}$ will denote the complex plane, $\hat{\mathbb{C}}$ the Riemann sphere and $E: \mathbb{C} \rightarrow \mathbb{C}$ a transcendental entire map.

Definition. A point $\omega \in \hat{C}$ is an asymptotic value for $E$ if there is a path $\alpha:[0,1) \rightarrow \mathbb{C}$ such that $\lim _{t \rightarrow 1} \alpha(t)=\infty$ and $\lim _{t \rightarrow 1} E \circ \alpha(t)=\omega$. The path $\alpha$ is a critical path for $\omega$.

Definitions. Let $\operatorname{AV}(E)$ be the set of asymptotic values of $E$; let $\operatorname{CV}(E)$ be the set of critical values of $E$ (images of critical points); let $\operatorname{SV}(E)=\operatorname{CV}(E) \cup \operatorname{AV}(E)$ be the set of singular values of $E$ (note that $\infty \in \mathrm{SV}(E)$ for any $E$ ); let $M_{E}=\hat{\mathbb{C}}-\overline{\operatorname{SV}(E)}$ ( $M_{E}$ is a manifold, it is possible that $M_{E}=\varnothing$ ); let $\tilde{M}_{E}=E^{-1}\left(M_{E}\right)$.

LEMMA 1.1. The map $E: \tilde{M}_{E} \rightarrow M_{E}$ is a covering.

Proof. Let $w \in M_{E}$, and choose a simply connected neighbourhood $U$ of $w$. Define $\varphi: E^{-1}(w) \times U \rightarrow E^{-1}(U)$ as follows. Let $z \in E^{-1}(w), u \in U$, and choose a simple path $\gamma$ in $U$ connecting $w$ to $u$. Since $w$ is not a critical value of $E$, we can define a local inverse $E_{z}^{-1}$ for $E$ on a neighbourhood of $w$, which is uniquely determined by the condition $E_{z}^{-1}(w)=z . U \cap \operatorname{SV}(E)=\varnothing$ so we can analytically continue $E_{z}^{-1}$ along $\gamma$ to $u$, and we define $\varphi(z, u)=E_{z}^{-1}(u)$. $\varphi$ is well defined by the monodromy theorem and since it is the inverse of a function, it is injective. It is easy to check that $\varphi$ is also surjective. It follows that $\varphi$ is a homeomorphism and that $E \mid \tilde{M}_{E}$ is a covering.

Let $V^{*}=V-E^{-1}(\omega)$. By lemma 1.1,E $\mid V^{*}: V^{*} \rightarrow U-\{\omega\}$ is a covering, therefore $V^{*}$ is homeomorphic to either an annulus, a punctured disk or a disk.

If $V^{*}$ is an annulus, then the bounded component of the complement either is mapped by $E$ to $\omega$, which can't happen since $E$ is locally injective, or contains a pole, which cannot happen since $E$ is entire. 
If $V^{*}$ is a punctured disk, then $E \mid V^{*}: V^{*} \rightarrow U-\{\omega\}$ is a finite to one cover, which can't happen since $V^{*}$ is unbounded while $U$ has compact closure.

Therefore, $V^{*}=V$ is homeomorphic to a disk, $E \mid V: V \rightarrow U-\{\omega\}$ is a covering and $\omega$ is logarithmic as claimed.

Proposition 1.3. If $E, F \in \Sigma, \operatorname{SV}(E \circ F)=\operatorname{SV}(E) \cup E(\operatorname{SV}(F))$.

COROLlARY 1.4. $\Sigma$ is closed under composition of functions.

Proof of 1.3. 1.3 clearly holds for critical values, (i.e. $\operatorname{CV}(E \circ F)=\operatorname{CV}(E) \cup$ $E(\mathrm{CV}(F)))$. It is also evident that $E(\operatorname{AV}(F)) \subset \operatorname{AV}(E \circ F)$. Suppose that $\omega \in \operatorname{AV}(E)$. By lemma 1.2, $\omega$ is logarithmic, so we can find a simply connected open neighbourhood $U$ of $\omega$ and a maximal, connected, unbounded component $V$ of $E^{-1}(U)$ such that $E \mid V: V \rightarrow U-\{\omega\}$ is a covering. In particular, $V \cap E^{-1}(\omega)=\varnothing$.

Definition. $E$ has finite type if $\operatorname{SV}(E)$ is a finite set. Let $\Sigma$ denote the collection of entire transcendental maps of finite type.

Examples. $z \rightarrow \sin z, z \rightarrow p(z) e^{z}$ where $p$ is a polynomial, $z \rightarrow e^{e^{z}}, z \rightarrow \int_{0}^{z} e^{-t^{n}} d t, n \geq 2$, belong to $\Sigma$.

It is our first goal to prove that $\Sigma$ is closed under composition. In order to do this, we need a technical result concerning the asymptotic values of $E \in \Sigma$.

Definition. $\omega \in \operatorname{AV}(E)$ is logarithmic if there is a simply connected neighbourhood $U, \omega \in U$, and a simply connected unbounded open set $V \subset E^{-1}(U)$ such that $E \mid V: V \rightarrow U-\{\omega\}$ is a covering.

For examples and a more general discussion of asymptotic values see [10, pp. 282289].

LEMMA 1.2. If $E \in \Sigma$ and $\omega \in \operatorname{AV}(E)$, then $\omega$ is logarithmic.

Proof. Since $\operatorname{SV}(E)$ is finite, we can find a simply connected neighbourhood $U$ of $\omega$ such that $U-\{\omega\} \cap \operatorname{SV}(E)=\varnothing$. Let $\alpha:[0,1) \rightarrow \mathbb{C}$, be a critical path for $\omega$ and let $V$ be the maximal connected component of $E^{-1}(U)$ containing $\alpha(t)$ for sufficiently large $t$.
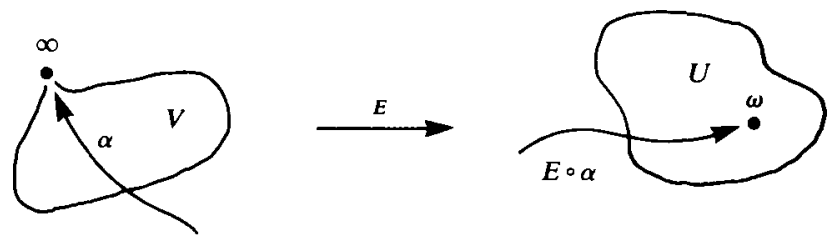

Choose any component $D$ of $F^{-1}(V)$ :

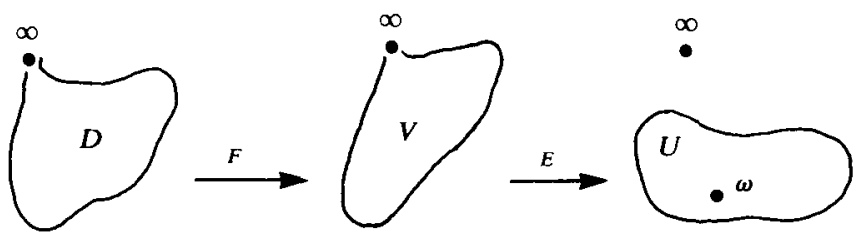


$D$ is an unbounded open set, and $D \cap(E \circ F)^{-1}(\omega)=\varnothing$. As in the proof of Iverson's theorem, [10], given any $\omega_{1} \neq \omega \in U$, and any $z \in(E \circ F)^{-1}(\omega)$, there is a path $\alpha:[0,1) \rightarrow D$ such that $\alpha(0)=z$, and $\lim _{t \rightarrow 1} \alpha(t)=\infty$ (i.e. $E \circ F \circ \alpha$ is a path connecting $\omega_{1}$ and $\omega$ which is completely contained in $U$ ). Therefore, $\omega$ is an asymptotic value of $E \circ F$, and we have shown $\operatorname{SV}(E) \cup E(\operatorname{SV}(F)) \subset \operatorname{SV}(E \circ F)$.

To show the opposite inclusion, let $\mathscr{B}=\mathbb{C}-[\operatorname{SV}(E) \cup E(\operatorname{SV}(F))]$. Since $E, F \in \Sigma$, we can find $w \in \mathscr{B}$ and a simply connected neighbourhood $U$ of $w, U \in \mathscr{B}$ such that $E^{-1}(U)=\bigcup V_{i}, V_{i} \cap V_{j}=\varnothing, i \neq j$, and $E \mid V_{i}: V_{i} \rightarrow U$ is a covering for each $i$ by lemma 1.1. Now fix $i$ and consider $F^{-1}\left(V_{i}\right)$. If $V_{i}$ contained points of $\operatorname{SV}(F), U$ would contain points of $E(\operatorname{SV}(F))$ and would not be entirely in $\mathscr{B}$. Again by lemma 1.1 $F^{-1}\left(V_{i}\right)=\bigcup W_{k}^{i}$ such that $F \mid W_{k}^{i}: W_{k}^{i} \rightarrow V_{i}$ is a covering for each $k$ and $W_{k}^{i} \cap$ $W_{l}^{i}=\varnothing, k \neq l$. It follows that $E \circ F$ is a covering onto $\mathscr{B}$ hence $\mathscr{B}$ contains no points of $\operatorname{SV}(E \circ F)$.

\section{Section 2}

In this section, we give definitions and technical constructions which will be used in $\S \S 3,4$.

Let $n \geq 3$, let $\zeta_{1}, \zeta_{2}, \ldots, \zeta_{n}$ be distinct points in $\hat{\mathbb{C}}$, and let $S=\hat{\mathbb{C}}-\left\{\zeta_{1}, \ldots, \zeta_{n}\right\}$. Define $T_{0}=\{f: \hat{\mathbb{C}} \rightarrow \hat{\mathbb{C}} \mid f$ is a quasiconformal homeomorphism $\}$, and let $T(S)=T_{0} / \sim$, where $f \sim g$ in $T_{0}$ if there is an analytic homeomorphism $h: \hat{\mathbb{C}} \rightarrow \hat{\mathbb{C}}$ such that $g^{-1} \circ h \circ f: \hat{\mathbb{C}} \rightarrow \hat{\mathbb{C}}$ is homotopic to the identity map rel $\left\{\zeta_{1}, \ldots, \zeta_{n}\right\}$.

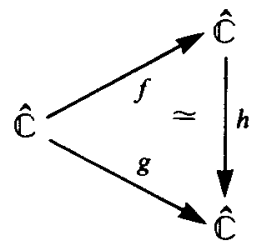

$T(S)$ is the Teichmüller space of $S$. A standard theorem is the following [1]:

THEOREM 2.1. $T(S)$ is canonically isomorphic to a domain in a complex vector space of dimension $n-3$.

The main object we consider in this paper is the space of entire functions quasiconformally conjugate to a given transcendental entire map $E$; namely, let

$\mathscr{T}(E)=\left\{F: \mathbb{C} \rightarrow \mathbb{C} \mid F\right.$ is entire, $F=f \circ E \circ f^{-1}$ where $f$ is quasiconformal $\}$.

For technical reasons, we need to consider covering spaces of $\mathscr{T}(E)$. Define

$$
T_{0}(E)=\left\{f: \mathbb{C} \rightarrow \mathbb{C} \mid f \text { is quasiconformal, } f \circ E \circ f^{-1} \text { is entire }\right\},
$$

and let $T(E)=T_{0}(E) / \sim$ where $f \sim g$ in $T_{0}(E)$ if:

(1) $f(p)=g(p)$ for all $p \in \mathrm{SV}(E)$;

(2) $f \circ E \circ f^{-1}=g \circ E \circ g^{-1}$;

(3) $f \circ g^{-1} \approx$ identity rel SV(E).

Remark 2.2. The quasiconformal maps $f$ such that $f \circ E \circ f^{-1}$ is entire are precisely the maps which are compatible with $E$. To be specific, let $\mu_{f}=f_{\bar{z}} / f_{z}$ where the 
derivatives are taken in the sense of distributions. $\mu_{f}$ is the Beltrami Coefficient of $f$, and satisfies the equation:

$$
\mu_{f}(E(z)) \cdot \overline{E^{\prime}(z)} / E^{\prime}(z)=\mu_{f}(z) \quad \text { a.e. }
$$

There is a projection $\pi: T(E) \rightarrow \mathscr{T}(E)$ defined by $\pi((f))=f \circ E \circ f^{-1}$.

LEMMA 2.3. The fibre of the projection $\pi, \pi^{-1}(F)$, is discrete.

Corollary. dimension $\mathscr{T}(E)=$ dimension $T(E)$.

Proof. An element $(f) \in T\left(M_{E}\right)$ induces an automorphism of the fundamental group $\varphi: \pi_{1}\left(M_{E}\right) \rightarrow \pi_{1}\left(M_{E}\right)$ which is defined up to inner automorphism. $\mathcal{M}=$ the group of automorphisms of $\pi_{1}\left(M_{E}\right)$ defined up to inner automorphisms $\}=$ the mapping class group of $M_{E}$, is known to act properly discontinuously on $T\left(M_{E}\right)$ (see [5], [8]). Consequently, the fibre of the projection $T\left(M_{E}\right) \rightarrow T\left(M_{E}\right) / \mathcal{M}$ is discrete.

Since the elements $(f) \in T(E)$ are compatible with $E$, not every element of $\mathcal{M}$ is induced by such an $(f)$. Let $\mathcal{M}_{0}=\{\varphi \in \mathscr{M} \mid \varphi$ is induced by $(f) \in T(E)\}$. $\mathcal{M}_{0}$ is a subgroup of $\mathcal{M}$ and so acts properly discontinuously on $T(E)$ for the same reasons $\mathcal{M}$ acts properly discontinuously on $T(E)$. It follows that the fibre of the projection $T(E) \rightarrow T(E) / \mathcal{M}_{0}$ is discrete.

\section{Section 3}

THEOREM 3.1. If $E$ has finite type, $E \in \Sigma$, then $T(E)$ and $\mathscr{T}(E)$ are finite dimensional spaces.

Proof. Case 1: $\mathrm{SV}(E)$ contains at least three points.

As in $\S 1$, let $M_{E}=\hat{\mathbb{C}}-\operatorname{SV}(E) . M_{E}$ is a sphere with $n \geq 3$ punctures and $T\left(M_{E}\right)$ is the Teichmüller space defined above. Define $\Phi_{0}: T_{0}(E) \rightarrow T\left(M_{E}\right)$ by

$$
\Phi_{0}\left(f \in T_{0}(E)\right)=\left[f: M_{E} \rightarrow f\left(M_{E}\right)\right] \in T\left(M_{E}\right) .
$$

$\Phi_{0}$ projects to a map $\Phi: T(E) \rightarrow T\left(M_{E}\right)$,

$$
\Phi((f) \in T(E))=[f] \in T\left(M_{E}\right),
$$

and we will show that the fibre of $\Phi$ has dimension at most four.

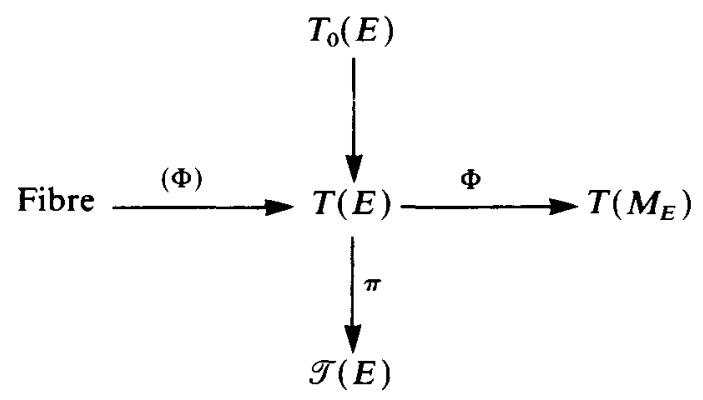

Suppose $(f) \in T(E)$ and $\Phi((f))=\left[\right.$ id]. Let $F=f \circ E \circ f^{-1}, M_{F}=f\left(M_{E}\right)$. Then there is an analytic homeomorphism $h: \hat{\mathbb{C}} \rightarrow \hat{\mathbb{C}}$ and a homotopy $H: \hat{\mathbb{C}} \times I \rightarrow \hat{\mathbb{C}} \operatorname{rel} \operatorname{SV}(E)$ such that $H(\cdot, 0)=f$ and $H(\cdot, 1)=h$. 


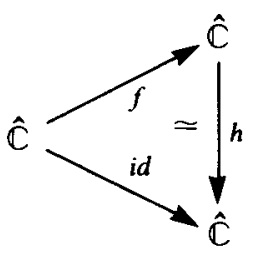

Recall $\tilde{M}_{E}=E^{-1}\left(M_{E}\right), \tilde{M}_{E}=F^{-1}\left(M_{F}\right) . H$ restricts to a homotopy $H: M_{E} \times I \rightarrow M_{F}$, and we consider the commutative diagram:

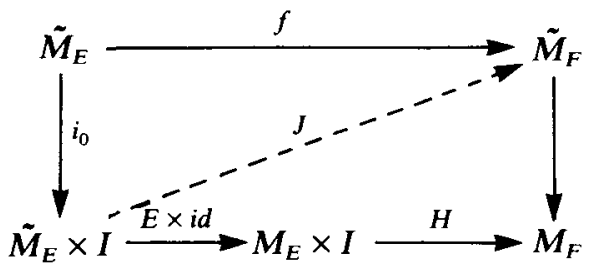

Since $F: \tilde{M}_{F} \rightarrow M_{F}$ is a covering by lemma 1.1 , the homotopy $H \circ E \times$ id lifts to a homotopy $J: \tilde{M}_{E} \times I \rightarrow \tilde{M}_{F}$ as drawn.

Let $j=J(\cdot, 1): \tilde{M}_{E} \rightarrow \tilde{M}_{F} \cdot j$ satisfies the functional equation:

$$
h \circ E=F \circ j \text {, }
$$

and is therefore analytic. We will show that $j$ extends to an analytic homeomorphism of $\mathbb{C}$ (i.e. an affine map) which satisfies $(*)$. It then follows immediately that $\Phi^{-1}([\mathrm{id}]) \subset\left\{(f) \in T(E) \mid \pi((f))=F=h \circ E \circ j^{-1}, h, j\right.$ affine $\} \approx \pi^{-1}(F) \times \mathbb{C}^{2}$. Since $\pi^{-1}(F)$ is discrete by lemma 2.3 , the theorem will be proved.

Let $a \in E^{-1}(\operatorname{SV}(E))=\mathbb{C}-\tilde{M}_{E}$. Since $\operatorname{SV}(E)$ is a finite set, $E^{-1}(\operatorname{SV}(E))$ is discrete, and we can find a simple closed curve $\gamma$ contained in $\tilde{M}_{E^{-1}}$ such that $\eta(\gamma, a)=1$, $\eta(\gamma, b)=0$ for all $b \in E^{-1}(\operatorname{SV}(E)), b \neq a$, where $\eta(\gamma, a)$ is the winding number of $\gamma$ with respect to $a$. Assume, without loss of generality, that $f$ is orientation preserving. Then $\eta(j(\gamma), f(a))=1$ and $\eta(j(\gamma), f(b))=0$. It follows that $j$ is bounded in the component of the complement of $\gamma$ containing $a$, and that $a$ is a removable singularity. Therefore, $j(a)=(1 / 2 \pi i) \int_{\gamma}(j(s) /(s-a)) d s=f(a)$ is an analytic extension of $j$ to $a$ and $j$ extends to an entire map which satisfies $(*)$.

To show that $j$ is affine, note the foi. ving two points:

(1) $j$ has no critical points; differentiating (*), we see $\operatorname{CV}(j) \subset \mathrm{CV}(E) \subset$ $E^{-1}(\operatorname{SV}(E))$, but $j$ is unramified at each $a \in E^{-1}(\operatorname{SV}(E))$.

(2) There are at least two values for which $j$ has only one pre-image; since $\# \mathrm{SV}(E) \geq 3$, some $\omega \in \mathrm{SV}(E)$ has infinitely many distinct pre-images $\left\{b_{i}\right\}$, but $j^{-1}\left(b_{i}\right)$ contains only one point for all $i$.

(1) implies $j$ is either affine or transcendental, and (2) together with Picard's theorem implies $j$ cannot be transcendental.

Case 2. $\operatorname{SV}(E)$ contains 2 points.

Choose an arbitrary point $\omega \notin \operatorname{SV}(E)$ and let $\operatorname{SV}(E)^{*}=\operatorname{SV}(E) \cup\{\omega\}, M_{E}^{*}=$ $\hat{C}-\operatorname{SV}(E)^{*}$, and repeat case 1 . 


\section{Section 4}

In this section we consider some dynamical properties of our class $\Sigma$ of functions and prove a 'no wandering domain' theorem.

First, we recall some basic definitions.

Definition. A point $z \in \hat{\mathbb{C}}$ is stable for $E$ if is a neighbourhood $U$ of $z$ such that the iterates $\left\{E^{n} \mid U\right\}$ form a normal family. $Q_{E}=\{z \mid z$ is stable for $E\}$ is an open and completely invariant set. $J_{E}=\hat{C}-Q_{E}$ is the unstable set or Julia set of $E$. It is closed, completely invariant, and non-empty. If it is not all of $\hat{C}$, it is perfect and nowhere dense.

The stable region breaks up into a disjoint union of components which can be of two types:

Definition. A component $D \subset Q_{E}$ is eventually periodic if there exist integers $m, n \geq 0$ such that $E^{m+n}(D) \subset E^{n}(D)$. Otherwise, $D^{n} \cap D^{m}=\varnothing$ for all $n \neq m \geq 0$, where $D^{n}$ is the component containing $E^{n}(D)$, and $D$ is said to be wandering. $D$ is unramified if $\operatorname{SV}(E) \cap D^{n}=\varnothing$ for all $n \geq 0$.

Remark 4.1. (i) If $D$ is unramified, $D^{n}=E^{n}(D)$ for all $n \geq 0$.

(ii) If $D$ is a wandering domain, and $\operatorname{SV}(E)$ is finite, we can find $N \geq 0$ such that $D^{N}$ is an unramified wandering domain.

As an application of theorem 3.1 we prove:

THEOREM 4.2. If $E \in \Sigma$, then $Q_{E}$ contains no wandering domains.

We will show that, if $\operatorname{SV}(E)$ is finite, the existence of an unramified wandering domain $D$ implies that $\mathscr{T}(E)$ is an infinite dimensional space. This contradicts theorem 3.1. Our proof is based on an argument due to Sullivan [13] which shows that the stable set of a rational map contains no wandering domains. Bers [6] gives a modified proof of Sullivan's result and our proof is modelled on the Bers version.

Suppose $D \subset Q_{E}$ is an unramified wandering domain. $D$ is covered by the unit disk $U$, and according to lemma 1.1, $E: D^{n} \rightarrow D^{n+1}$ is a covering for all $n$. Therefore we have a commutative diagram:

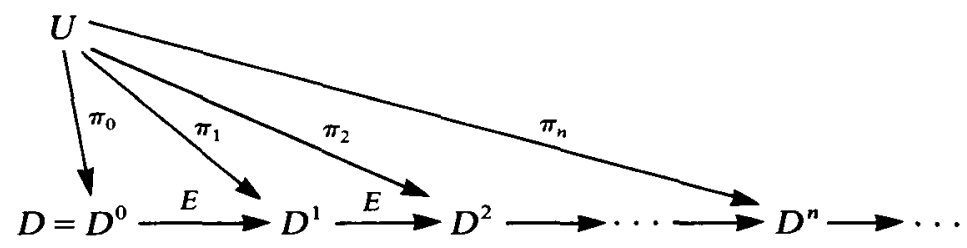

where $\pi_{n}: U \rightarrow D^{n}$ is a holomorphic universal covering map. Let $\Gamma^{n}$ denote the fundamental group of $D^{n}$, then $\Gamma^{0} \subset \Gamma^{1} \subset \cdots$ is an increasing sequence of discrete groups.

Proposition 4.3. If $\Gamma^{n}$ is trivial for all $n$ then $\mathscr{T}(E)$ has infinite dimension.

Proposition 4.4. If $E \in \Sigma$ and $D$ is an unramified wandering domain, then $\Gamma^{n}$ is trivial for all $n$.

These two propositions, together with remark 4.1 and theorem 3.1, imply theorem 4.2. 
We will now prove a technical result (lemma 4.9) which is the basis of the proof of proposition 4.3. Let $D \subset \hat{C}$ be an open set.

Definition. A Beltrami Coefficient in $D$ is an element $\mu \in L_{\infty}(D)$ such that $\|\mu\|_{\infty}<1$.

Suppose $\partial D$ contains at least 3 points. Then $D$ admits a metric of constant curvature -1 , which we denote by $\rho_{D}(z)|d z|$.

Definition. A Beltrami coefficient $\mu$ in $D$ is harmonic if $\mu(z)=\rho_{D}(z)^{-2} \overline{\psi(z)}$ where $\psi$ is holomorphic.

Let $\mathscr{H}(D)$ denote the space of harmonic Beltrami coefficients in $D$.

THEOREM 4.5. Let $D \subset \hat{\mathbb{C}}$ be open. Suppose either (i) $\partial D$ has non-empty interior or (ii) $\pi_{1}(D)$ is not finitely generated. Then $\mathscr{H}(D)$ is an infinite dimensional space.

Proof. This is a special case of [6, lemma 4.1].

Remark 4.6. If $D \subset \hat{\mathbb{C}}$ is a wandering component of the stable region of a rational of entire map, than $D$ satisfies (i) above.

Definition. A Beltrami coefficient $\mu$ in $\mathbb{C}$ is eventually harmonic for $E: \mathbb{C} \rightarrow \mathbb{C}$ if:

(1) $\mu(z)=\mu(E(z)) \cdot \overline{E^{\prime}(z)} / E^{\prime}(z)$ [see remark 2.2];

(2) for each component $D \subset Q_{E}$, there is an $N>0$ such that, for all $n \geq N, \mu \mid D^{n}$ is harmonic.

Let $\mathscr{L}_{E}(\mathbb{C})$ denote the space of eventually harmonic Beltrami coefficients in $\mathbb{C}$ for $E$. From the measurable Reimann mapping theorem [2] we obtain a holomorphic map $\theta: \mathscr{H}_{E}(\mathbb{C}) \rightarrow \mathscr{T}(E)$ as follows:

To each $\mu \in \mathscr{H}_{E}(\mathbb{C})$, there is a unique normalized quasiconformal homeomorphism $f^{\mu}: \mathbb{C} \rightarrow \mathbb{C}$, fixing 0 and 1 such that $f^{\mu} \circ E \circ\left(f^{\mu}\right)^{-1}$ is entire. We define $\theta(\mu)=$ $f^{\mu} \circ E \circ\left(f^{\mu}\right)^{-1} \in \mathscr{T}(E)$.

LEMMA 4.7. The fibre of $\theta$ over any point $F \in \mathscr{T}(E)$ is a discrete set.

Proof. The proof of lemma 4.7 is identical to the proof of lemma 7.1 in [6], choosing $d+1$ eventually harmonic differentials, where $d=$ dimension $\mathscr{T}(E)$, and replacing everywhere, the rational map ' $R$ ' with the entire map ' $E$ '.

The following is immediate.

Corollary 4.8. dimension $\mathscr{H}_{E}(\mathbb{C})=$ dimension $\mathscr{T}(E)<\infty$.

LEMMA 4.9. Suppose $D$ is an unramified wandering domain for a rational or entire map $E$, and there is an $N$ such that $E: D^{n} \rightarrow D^{n+1}$ is injective for all $n \geq N$. Then $\mathscr{H}_{E}(\mathbb{C})$ is infinite dimensional.

Proof. To simplify notation, set $D=D^{N}$. Since $D$ is unramified, $E$ is a bijection, and we can define $G^{n}=\left(E^{n} \mid D\right)^{-1}, n=0,1, \ldots$ By theorem 4.5 and remark 4.6 there are infinitely many linearly independent harmonic Beltrami coefficients $\mu_{1}, \mu_{2}, \ldots$ in $D$. For $z \in D^{n}, n \geq 0$, set

$$
\hat{\mu}_{j}(z)=\mu_{j}\left(G^{n}(z)\right) \frac{\overline{\left(G^{n}\right)^{\prime}(z)}}{\left(G^{n}\right)^{\prime}(z)},
$$


and if $E^{m}(z) \in E^{n}(D), m \geq 0, n \geq 0$, set

$$
\hat{\mu}_{j}(z)=\hat{\mu}_{j}\left(E^{m}(z)\right) \frac{\overline{\left(E^{m}\right)^{\prime}(z)}}{\left(E^{m}\right)^{\prime}(z)} .
$$

For all other $z$, set $\hat{\mu}_{j}(z)=0 . \hat{\mu}_{1}, \hat{\mu}_{2}, \ldots$ are well defined, and form an infinite set of linearly independent Beltrami coefficients compatible with, and eventually harmonic with respect to $E$.

Proof of proposition 4.3. By assumption, each $D^{n}$ is homeomorphic to a disk. Furthermore, $E: D^{n} \rightarrow D^{n+1}$ is an unramified covering for all $n$, and so is a bijection. Proposition 4.3 is therefore a direct consequence of lemma 4.9.

In order to prove proposition 4.4 , we will need two facts about entire maps.

LEMMA 4.11 (Tangerman [14]). Suppose $E \in \Sigma$, and let $\Delta \supset \operatorname{SV}(E)$ be a set which is homeomorphic to a closed disk. Then every connected component of $E^{-1}(\mathbb{C}-\Delta)$ is unbounded and homeomorphic to a disk.

Proof. Let $V$ be a component of $E^{-1}(\mathbb{C}-\Delta)$. Then $E \mid V: V \rightarrow \mathbb{C}-\Delta$ is a covering by lemma 1.1. Hence, $(E \mid V)_{*}: \pi_{1}(V) \rightarrow \pi_{1}(\mathbb{C}-\Delta) \cong \mathbb{Z}$ is injective, so that $\pi_{1}(V) \cong \mathbb{Z}$ or $\pi_{1}(V)$ is trivial. In the former case, $V$ is homeomorphic to either a punctured disk or an annulus. If $V$ is homeomorphic to a punctured disk, then $E$ must have a pole at the puncture, which cannot happen since $E$ is entire. If $V$ is homeomorphic to an annulus, $E$ must map the inner boundary of $V$ to $\infty$, which does not make sense since $E$ is entire. Consequently, $\pi_{1}(V)$ is trivial, and $V$ is homeomorphic to a disk.

TheOREM 4.11 [4, theorem 3.1]. (1) A multiply connected component $D$ of the stable set of a transcendental entire function $E$ is wandering.

(2) On $D$, every sequence $\left\{E^{n}\right\}$ of iterates $E$ contains a subsequence that converges uniformly to $\infty$ on every compact subset of $D$.

(3) For large $n, E^{n}(D)$ contains a smooth closed curve $\gamma^{n}$, whose winding number about the origin is non-zero, and whose distance from the origin increases to $\infty$ as $n$ goes to $\infty$.

(4) Moreover, every component of the stable region is bounded.

Proof. See [4, theorem 3.1].

Proof of proposition 4.4. Suppose for some $N, \Gamma^{n}$ is non-trivial for all $n \geq N$. Without loss of generality, assume $N=0$. Let $\gamma_{0}$ be a smooth representative for a generator of $\Gamma^{0}, \gamma_{n}=E^{n}\left(\gamma_{0}\right)$, and let $B^{n}$ denote the bounded component of the complement of $\gamma^{n}$. By theorem 4.11, we can choose $n$ so large that $\mathrm{SV}(E) \subset B^{n}$. By lemma 4.10, each connected component of $E^{-1}\left(\gamma^{n}\right)$ is unbounded (and a closed submanifold of $\mathbb{C}$ ). Since $D^{n-1}$ is bounded by theorem 4.11 , and contains one of these pre-images, we have a contradiction.

\section{REFERENCES}

[1] L. V. Ahlfors. Lectures on Quasiconformal Mappings. Van Nostrand, 1966.

[2] L. V. Ahlfors \& L. Bers. Riemann's mapping theorem for variable metrics. Ann. of Math. 72 (1960), 385-404. 
[3] I. N. Baker. The domains of normality of an entire function. Ann. Acad. Sci. Fenn. Ser. A.I. Math. 1 (1975), 277-283.

[4] I. N. Baker. Wandering domains in the iteration of entire functions. Preprint, 1983.

[5] L. Bers. Fiber spaces over Teichmüller spaces. Acta Math. 130 (1973), 89-126.

[6] L. Bers. On Sullivan's proof of the finiteness theorem and the eventual periodicity theorem. Preprint.

[7] P. Fatou. Sur l'itération des fonctions transcendentes entières. Acta math. 47 (1920), 337-370.

[8] R. Fricke \& F. Klein. Vorlesungen über die Theorie der Automorphen Funktionen, Vol. 1, part II.2; reprinted 1965, Academic Press.

[9] L. Goldberg \& L. Keen. On stable regions for a class of entire functions. In preparation.

[10] R. Nevanlinna. Analytic Functions. Springer Verlag, 1970.

[11] J. Nielsen. Untersuchung zur Topologie der geschlossen zweiseitigen Flächen. Acta math. 501927.

[12] C. L. Seigel. Bermerkung zu einem Satz von Jakob Nielsen. In Gesammelte Abhandlungen. SpringerVerlag, 1966, Vol. 3, pp. 92, 96.

[13] D. Sullivan. Quasiconformal homeomorphisms and dynamics I. Preprint.

[14] F. Tangerman. Personal communication. 